\title{
Gouvernance de l'effectif et de la répartition des médecins
}

Depuis quelques années, il est question de pénurie de professionnels de la santé en Suisse. En témoignent d'une part les difficultés rencontrées pour pourvoir les postes vacants dans les hôpitaux et, d'autre part, le nombre élevé de professionnels de la santé avec un diplôme obtenu dans un pays étranger. Face à cette situation, le Conseil fédéral a planifié diverses mesures dans le cadre de l'initiative visant à combattre la pénurie de personnel qualifié dans le domaine de la santé. Le message FRI 2017-20 prévoit en outre CHF 100 millions pour la création de places de formation supplémentaires pour les médecins. Quelle est l'influence du nombre de médecins sur l'ensemble du système de santé? Quelles sont les possibilités et les limites de la gouvernance? L'ASSM soulève ces questions dans une nouvelle feuille de route.

En 2012, l'ASSM avait déjà mentionné dans sa feuille de route «Médecine durable» qu'en Suisse la pérennité de la médecine respectivement du système de santé n'était pas assurée. Un système de santé durable suppose également un nombre adéquat de médecins et de professionnels de la santé ainsi qu'une répartition géographique et professionnelle conforme aux besoins. Avec 4,0 médecins respectivement 17,4 infirmiers par 1000 habitants (2013), la Suisse a une des densités de professionnels de la santé les plus élevées au monde. $\mathrm{Au}$ niveau international, les expériences ont montré que, dans un système de santé financé solidairement, la gouvernance des ressources humaines et notamment des ressources médicales était incontournable. Les médecins sont les acteurs clés, car ils détiennent le monopole en matière de diagnostic et d'indication médicale. Le système de santé est un système extrêmement complexe, c'est pourquoi une telle gouvernance doit être réalisée pas à pas, c'est-à-dire en combinant divers instruments tout en impliquant les principaux partenaires.

Dans sa nouvelle feuille de route, l'ASSM analyse la situation à l'exemple des médecins, montre les possibilités et les limites de la gouvernance et formule des recommandations à l'attention des différents acteurs du système de santé. La feuille de route, dont des extraits sont reproduits ici, s'adresse aux nombreux acteurs du système de santé, entre autres aux fournis- seurs de prestations, aux politiciens de la santé aux niveaux cantonal et fédéral, aux bailleurs de fonds et aux établissements d'enseignement. L'ASSM est consciente qu'actuellement de nombreux projets sur ce thème sont en cours. L'objectif prioritaire de cette feuille de route est de renforcer leur action.

\section{Interprétation large de la notion de gouvernance}

Le problème des mauvaises répartitions des ressources médicales et leurs conséquences est analysé et discuté depuis des années, tant en Suisse qu'à l'étranger. Ces analyses et discussions se déroulent dans des contextes très différents, avec des répercussions sur la prise en charge correspondante, son cadre politique ou les profils des acteurs. Dès lors, il n'est pas surprenant que les initiatives et les mesures adoptées par les différents pays soient également différentes. On peut néanmoins $\mathrm{y}$ trouver un dénominateur commun: dans un système de santé financé solidairement, la gestion des ressources médicales est indispensable.

Le terme de "gouvernance» doit être interprété au sens large; il ne comprend pas uniquement des mesures

\section{Indications concernant l'élaboration}

\section{de cette feuille de route}

Les personnes suivantes font partie du groupe de travail qui a élaboré cette feuille de route: Prof. Urs Brügger, Winterthour (Direction); Dr Hermann Amstad, Berne; PD Dr Peter Berchtold, Berne; Prof. Iren Bischofberger, Zurich; Prof. Peter Meier-Abt, Zurich; Prof. Arnaud Perrier, Genève; Prof. Martin Schwab, Zurich; Prof. Peter Suter, Presinge; Prof. Martin Täuber, Berne; Prof. Thomas Zeltner, Berne.

Le groupe de travail s'est réuni à plusieurs reprises pour réfléchir à cette thématique. Après une étude de la littérature et des discussions approfondies, il a rédigé un premier projet de ce document et l'a ensuite soumis au Comité de direction de l'ASSM et à des experts: Dr Werner Bauer, Président ISFM, Küsnacht; Prof. Manuela Eicher, Institut universitaire de formation et de recherche en soins, Université de Lausanne; Prof. Martina Merz, FHS St-Gall; lic. rer. pol. Michael Jordi, Secrétaire central CDS, Berne; Dr Anja Zyska Cherix, Comité de direction VSAO, Lausanne. Le Comité de direction de I'ASSM a discuté et approuvé la version finale lors de sa séance du 5 septembre 2016. 
fixées par l'État. Il englobe également la régulation des incitations (financières ou autres). Ce faisant, il ne faut pas oublier que le système de santé est extrêmement complexe - et que les médecins n'en sont qu'une partie. Dès lors, les répercussions des mesures de gouvernance dans un domaine partiel du système sont difficilement prévisibles. Il importe de tenir compte de ces réalités en cas d'intervention dans la gestion du système.

\section{Recommandations}

L'ASSM propose cinq groupes de mesures pour la gouvernance de l'effectif et de la répartition des médecins dans le système de santé suisse. La prise de conscience de la nécessité d'adopter des mesures combinées les unes aux autres est au cœur de ces recommandations; loin de produire les effets escomptés, les interventions de gouvernance isolées provoquent surtout des effets secondaires indésirables. Pour chaque mesure, il est indiqué avec quelle priorité elle doit être adoptée et si elle doit être réalisée à court ou à moyen terme. Certaines de ces recommandations ont déjà été formulées par l'ASSM dans un autre contexte (médecine durable; futurs profils professionnels); elles gardent leur validité.

\section{Dans un système de santé financé solidaire- ment, la gestion des ressources médicales est indispensable.}

Pour une gouvernance réussie, il importe en premier lieu de prendre en compte les nombreux facteurs qui influencent l'offre en prestations médicales. Deuxièmement ces mesures doivent être envisagées et implémentées dans le cadre d'un dialogue entre les acteurs du système de santé, les bailleurs de fonds et les régulateurs. La plateforme «Avenir de la formation médicale» offre à cet égard une première approche. Comme en témoignent les expériences internationales, la correction trop lente et insuffisante de la mauvaise répartition géographique et professionnelle peut, en partie, être attribuée au manque de coordination des nombreux acteurs dans la planification du besoin médical.

\section{Conception d'un système d'incitation cohérent} Dans le choix de la discipline comme du lieu de travail, de nombreux facteurs, financiers ou non, jouent un rôle. C'est pourquoi il importe de concevoir des systèmes d'incitations multidimensionnels. De tels modèles associent des incitations extrinsèques (avant tout financières) et des aspects intrinsèques (par exemple organisation du travail, assistance, réseau) d'une manière cohérente (c'est-à-dire complémentaire).
Compte tenu des déficiences sérieuses dans les systèmes tarifaires, relatives à la mauvaise répartition des ressources médicales, des incitations financières cohérentes ne peuvent être réalisées qu'avec une révision et une optimisation des tarifs des prestations médicales. A cet égard, l'attention sera essentiellement portée sur

1) la révision du TARMED telle qu'elle est actuellement planifiée avec la réduction de certains tarifs de spécialistes et la revalorisation des prestations de médecine de premier recours (médecine générale, psychiatrie, pédiatrie),

2) des programmes return-of-service pour les zones rurales, et

3) des modèles de remboursement liés aux performances.

\section{Création d'une base de données}

L'analyse et la prévision des besoins sont au cœur de toutes les interventions de gouvernance; ces données sont indispensables pour définir quelles sont les qualifications et les offres médicales pouvant répondre aux différentes demandes de prestations médicales. «Il est difficile de faire des pronostics, surtout quand ils concernent l'avenir» - cette affirmation s'applique également à ce contexte. Ainsi, on peut conclure de manière laconique, mais importante, que les pronostics à court terme donnent des résultats plus fiables. Dès lors, il importe d'observer continuellement l'évolution du personnel spécialisé, dans le sens d'un système apprenant, et de n'établir des pronostics que pour des courtes périodes. Des discussions approfondies sur l'élaboration d'un concept de gouvernance n'étant guère pertinentes sans une telle base de données, un modèle de calcul concernant les besoins médicaux devrait être élaboré sans attendre et les premiers calculs devraient être effectués, même si les informations à disposition sont encore incomplètes. Comme mentionné ci-dessus, l'Obsan élabore actuellement un tel modèle de calcul; il s'agit maintenant de vérifier ce modèle, de l'ajuster et de l'utiliser comme base de la gouvernance.

\section{Développement d'un système de gouvernance supracantonal efficace}

Les interventions de gouvernance concernent de nombreux acteurs du système de santé et, donc, un large éventail de rôles, de responsabilités et d'intérêts. Il va de soi que ces intérêts sont divergents, voire quelquefois contradictoires. L'efficacité des projets de gouvernance dépend fortement de la mesure dans laquelle les différents intérêts et exigences peuvent être harmonisés, mais également de la possibilité d'élaborer ensemble et d'adopter des mesures soutenues par tous les partenaires. 
Un système de gouvernance avec une participation effective des acteurs est la condition préalable à ce nouveau processus de concertation et de décision. Celui-ci comprend 1) des institutions et des organes dont le rôle, les tâches et les responsabilités sont claires, 2) des processus de travail et de décision harmonisés entre eux, 3) des recommandations au caractère contraignant, et 4) une évaluation des résultats. A cet égard, le groupe thématique "Coordination de la formation postgraduée médicale» de la plateforme «Avenir de la formation médicale» a d'ores et déjà élaboré les premières propositions.

\section{L'augmentation des places de formation, à elle seule, ne suffit pas à sortir la prise en charge médicale de l'impasse.}

Le système de gouvernance et les trains de mesures doivent tenir compte du système de soins fédéraliste. De même, pour une gouvernance réussie de l'effectif et de la répartition des médecins, un nouveau niveau de gouvernance supracantonale pourrait s'avérer nécessaire. Ce niveau compterait environ sept régions (par analogie aux régions des hautes écoles spécialisées) avec une population d'au moins 1 million d'individus. Au sein de ces régions, l'offre médicale pourrait être gérée beaucoup plus efficacement que dans les cantons qui présentent d'énormes différences.

\section{Modulation de la formation pré- et postgraduée}

Les CHF 100 millions alloués pour la création de places de formation supplémentaires pour les médecins, dans le cadre du message FRI 2017-20, devraient permettre entre autres d'augmenter les effectifs de la relève en médecine de premier recours et de réduire la dépendance aux médecins avec un diplôme étranger. Le niveau master des études en médecine est particulièrement important pour les futurs médecins et pour leur activité professionnelle, car environ la moitié des médecins prennent les décisions liées à leur carrière à ce moment-là. Cette période représente un tournant déci- sif pour l'exercice futur de la profession. Les exigences du message FRI 2017-20 ne pourront être satisfaites que si la formation médicale est plus nuancée et qu'elle accorde plus de poids aux aspects liés à l'interprofessionnalité et à l'interdisciplinarité dès les études. De tels modèles ont fait leurs preuves à l'étranger et sont également envisagés en différents endroits en Suisse (EPF, Fribourg, St-Gall, Lucerne, Tessin).

L'augmentation des places de formation, à elle seule, ne suffit pas à sortir la prise en charge médicale de l'impasse. Un engagement dans la formation postgraduée des médecins est également nécessaire. Des mesures supplémentaires doivent être prises dans cette phase, pour limiter autant que possible la perte de professionnels qualifiés. Il s'agit de tenir davantage compte des valeurs et des idées des jeunes générations, pour permettre notamment aux femmes de concilier à long terme les objectifs de vie et les buts professionnels. Les femmes constituant d'ores et déjà la majorité des professionnels de la santé, chaque réforme doit tenir compte de cette réalité.

\section{Soutien de nouveaux modèles de prise en charge}

Les nouveaux modèles de soins permettent d'améliorer la collaboration entre les différents groupes professionnels et d'optimiser la répartition du travail et des compétences grâce à des réseaux et des parcours de patients bien définis et organisés. Outre les améliorations au niveau de l'affectation des ressources, l'attractivité des professions peut ainsi être augmentée. Les nouveaux modèles de soins doivent prévoir des conditions de travail modernes et attractives pour les actuels et futurs professionnels de la santé. Les mesures formulées dans la feuille de route «Médecine durable» n'ont rien perdu de leur actualité.

\footnotetext{
Les références sont mentionnées dans la feuille de route. Celleci peut être commandée gratuitement au secrétariat général de I'ASSM ou téléchargée sur le site Internet: www.assm.ch $\rightarrow$ Publications $\rightarrow$ Feuilles de route
} 